\title{
A delayed modulation of solar radiation on the COVID-19 transmission reflects an incubation period
}

\author{
Maosheng He ${ }^{\circledR 1, \dagger}$, Keyan Fang ${ }^{\circledR 2,3, *, \dagger}$, Feifei Zhou ${ }^{2}$, Tinghai $\mathrm{Ou}^{3}$, and Deliang Chen ${ }^{\circledR} 3$ \\ ${ }^{1}$ The department of Physics and Earth Sciences, Jacobs University Bremen, Campus Ring 1, 28759 Bremen, \\ Germany \\ ${ }^{2}$ Key Laboratory of Humid Subtropical Eco-geographical Process (Ministry of Education), Fujian Normal University, \\ Fuzhou 350007, China \\ ${ }^{3}$ Regional Climate Group, Department of Earth Sciences, University of Gothenburg, Gothenburg 460, Sweden \\ *Communication to kujanfang@gmail.com \\ $\dagger$ these authors contributed equally to this work
}

\begin{abstract}
Laboratory experiments have revealed the meteorological sensitivity of the virus of the coronavirus disease 2019 (COVID19). However, no consensus has been reached about how the meteorological conditions modulate the virus transmission as it is constrained more often by non-meteorological factors. Here, we find that the non-meteorological factors constrain statistically-least the growth rate of cumulative confirmed cases in a country when the cases arrive around 2500-3000. The least-constrained growth rate correlates with the near-surface ultraviolet flux and temperature significantly (correlation coefficients $r=-0.55 \pm 0.08$ and $-0.45 \pm 0.08$ at $p<10^{-5}$, respectively). In response to increases of $1 \mathrm{~W} / \mathrm{m}^{2}$ ultraviolet and $1^{\circ} \mathrm{C}$ temperature, the growth rate decreases by $0.33 \pm 0.11 \%$ and $0.18 \pm 0.08 \%$ per day, respectively. The response to the ultraviolet flux exhibits a delay by about 7 days, providing an independent measure of the incubation period. Our quantifications imply a seasonality of COVID-19 and a high risk of a pandemic resurgence in the upcoming boreal winter, suggesting a need for seasonal adaption in public policies.
\end{abstract}




\section{Introduction}

It has attracted extensive attention whether the coronavirus disease 2019 (COVID-19) will spread more in the coming winter. Winters are often accompanied by peaks of virus transmissions, such as some Human coronaviruses ${ }^{1}$ and influenza ${ }^{2}$. These seasonalities are typically explained in terms of modulations of meteorological conditions on the virus survival, e.g., ${ }^{3}$. This knowledge has raised fears that COVID-19 may surge when the seasons change.

A way to predict the COVID-19 transmissions in the winter is to investigate the responses of the virus survival and transmission to the winter weather conditions. Laboratory experiments revealed that the ultraviolet radiation and air temperature modulates the airborne survival of the virus strongly ${ }^{4-6}$. Several studies have attempted to extract epidemiological evidence, e.g. ${ }^{7-10}$, through studying the correlations between the number of infections or mortality and the corresponding meteorological conditions using data from priorly selected cities. Their conclusions, however, are controversial. Some attempts did not find the correlation, e.g., ${ }^{6,7}$, whereas the others reported weak or moderate correlations, e.g., ${ }^{8-10}$. The results are not conclusive, mainly because the meteorological modulations are constrained by non-meteorological effects. In the current work, we identify the least-constrained growth rate of the infection and quantify its response to meteorological conditions statistically.

\section{Results}

An uncontrolled outbreak of COVID-19, e.g., ${ }^{11}$, starts with an exponential growth, the rate of which is presumably modulated by external factors such as weather ${ }^{4-6}$. We estimate the growth rate through a sliding window regression for each country and investigate its response to meteorological conditions across all countries, in contrast to the above-mentioned works ${ }^{7-10}$, which analyzed the number of confirmed cases or mortality from prior selected cities directly. Therefore, our results allow capturing straightforward the modulation of the weather and are not subject to potential statistical bias.

\section{The least-constrained growth rate}

Ideally, outbreaks of infectious diseases e.g. ${ }^{11-13}$, are typically characterized by two phases with the uncontrolled first phase showing stable exponential growth followed by a second phase with a decreasing growth rate usually after effective artificial controllers $^{14,15}$ (as sketched by the green lines in Figures S1b and S2a). The realistic evolution of the regressed growth rate of confirmed cases decreases largely with time, which can be divided into three primary stages (Figure S1 as an example and Figure S2 for all countries). In the beginning, infections cannot be confirmed timely and can accumulate until sufficient tests, which is different from the uncontrolled ideal model. Therefore, in Phase I, the decreasing growth rate reflects more the improvement of the capability than the infection growth. In Phase III, the decreasing growth rate could be explained as responses to artificial interventions or controllers, e.g., travel restrictions and changes in human behaviors, e.g., ${ }^{16,17}$. Also, at the late stage, cured cases are not infectious anymore and therefore lower the growth rate. Phase III corresponds to the decline stage of the ideal evolution model.

Therefore, it is most likely in Phase II, in-between the Phases I and III, where the uncontrolled transmission of COVID19 is recorded timely and sufficiently. Phase II is characterized by a relatively stable growth rate (Figures S1b and S2a), reflecting presumably the most modulation from the weather. In practical, instead of delimiting definitely the stage II for each country, we determine the most stage-II-representative growth rate, referred hereafter as least-constrained growth rate, for all countries through a single-parameter optimization (see "Methods"). The optimization maximizes the correlation between the least-constrained growth rate and meteorological variables. We obtain the solution of the optimization when the accumulative confirmed cases arrive statistically around 2500-3000 (in Phase II in Figure S2a).

\section{Impact of meteorological factors on the growth rate}

In Figure 1, Africa, central Asia and Central America are generally associated with low least-constrained growth rates, whereas high growth rates are observed in middle and high latitudes of North America and Europe. The obvious spatial clusters of the growth rate suggest its potential links to meteorological conditions. The growth rate exhibits significant correlation with the ultraviolet (UV) flux and the air temperature $(r=-0.55 \pm 0.08$ and $-0.45 \pm 0.08$ at $p<<0.01$, in Figures $2 \mathrm{a}$ and $2 \mathrm{~b}$, respectively), but not with the other meteorological conditions, namely, wind speed, relative humidity, diurnal temperature range, and precipitation ( $p>=0.05$, Figures 2c-2f). The regressions (in red in Figures 2a and $2 b$ ) quantify the responses. An increase in UV flux by $1 \mathrm{~W} / \mathrm{m}^{2}$ is associated with a decrease in the growth rate by $0.33 \pm 0.11 \%$ per day, and an increase of the temperature by $1^{\circ} \mathrm{C}$ is associated with a decrease in the growth rate by $0.18 \pm 0.08 \%$ per day.

\section{A measure of the incubation period}

Note that the above correlation and regression analyses involve a time displacement of 7 days between the meteorological variables and the growth rate. The correlation between the growth rate and the UV flux (but not the temperature) weakens suddenly as the time displacement decreases when the displacement is shorter than 7 days (Figures 3 ). The displacement 
indicates the presence of an incubation period as revealed previously ${ }^{18,19}$. The clinical study ${ }^{19}$ suggests that the incubation does not follow a normal distribution but is characterized by a positive skew (more cases occurred below the mean) with a median of 5.1 days and a mean of 6.4 days, whereas a cross-sectional and forward follow-up analysis ${ }^{18}$ reported a median incubation of 7.76 days. The discrepancy might be due to sampling bias in the forward follow-up approach which is less capable of detecting incubations shorter than four days. Similarly to the forward follow-up approach, our sliding window in the cross-correlation approach (Figure 3) captures the cases with incubation periods over the most frequency value, and therefore our diagnosis is consistent more with the forward follow-up analysis. Our results provide independent evidence of the incubation period by correlations from all countries, using approaches completely different from the existing estimations.

\section{Discussion}

In "Results", we illustrate that the least-constrained growth rate exhibits obvious spatial clusters and significant correlation with the meteorological conditions, namely, UV flux and temperature. The UV correlation exhibits a delay of about seven days, at the temporal scale of the incubation period. While the spatial clusters and the correlation might be attributed to the spatial difference of socioeconomic factors ${ }^{20,21}$, the delay cannot. The variations of socioeconomic factors are overall at temporal scales much longer than that of the delay, which can neither modulate the COVID-19 nor respond to the UV flux at the time scale of the incubation period.

To explain the correlation, in the current section, we explore potential causalities between the UV flux and the growth rate.There are at least three factors through which meteorological conditions can modulate the transmission. The first is human behaviors. When the temperature is low, humans typically spend more time indoors, with reduced social distancing and less ventilation than outdoors. As an example, schools are places of enhanced influenza transmission ${ }^{22}$ for intense indoor activities. The second factor is the immune system of susceptible hosts. Solar radiation drives changes in the human immune system by modulating melatonin ${ }^{23}$ and/or vitamin $\mathrm{D}^{24-26}$.

The last but might be the most important factor is the survival of the virus, namely the virucidal effect of UV. Evidence has revealed that the aerosols as a medium of transmission of COVID-19, as the virus remains active on the surfaces for several hours to days ${ }^{14}$. Intense solar radiation may inactivate the virus on the surface through the physical properties (i.e., shape, size) and the genetic material of the virus ${ }^{5,27,28}$. Simulation results suggested that $90 \%$ of the virus can be inactive under summer daytime for 6 minutes, whereas the virus becomes inactive for 125 minutes under night condition ${ }^{4}$. In addition, high temperature shortens the virus survival ${ }^{6,29,30}$. On the opposite, low temperature is in favor of prolonging survival on infected surfaces and aerosols, which promotes the diffusion of the infection. The modulation of relative humidity, on the other hand, is negligible, as supported by laboratory experiments ${ }^{4}$, which is different from the sensitive modulation on the influenza virus survival $^{3}$ and transmission ${ }^{31}$.

The 7-day-delayed response to the UV flux (Figure 3) reflects the incubation period, whereas the response to the temperature does not exhibit a delay. A potential scenario is that temperature variation is characterized by a temporal scale longer than the incubation period, and therefore cannot resolve the incubation period. Another potential scenario is that the temperature might not be an independent driver of the transmission but a response to solar radiation. The temperature correlates significantly with the UV flux $\left(r=0.78 \pm 0.05\right.$ at $\left.p=5.8 \times 10^{-22}\right)$. We carried out a canonical-correlation analysis, e.g. ${ }^{32}$, between the growth rate and the UV flux and temperature, resulting in a canonical correlation coefficient $c_{U V, T}=-0.56 \pm 0.08$. The canonical correlation coefficient is close to the correlation of UV $r_{U V}=-0.55 \pm 0.08$ (Figure 2a), which implicates that using both the UV and temperature as predictors can not explain more variance of the growth rate than using the UV alone.

The dominant impact of the UV flux can drive a seasonality of COVID-19 transmission and explain the following geographic dependence of COVID-19. (1) The mortality exhibits a latitudinal dependence ${ }^{26}$. (2) The late outbreak in Africa and arid central Asia is attributable to intense UV flux due to the low cloud fraction prior. (3) The onset of the Asian summer monsoon, increases clouds in early May ${ }^{33}$ and yields low UV flux, which may account for the late outbreak in India and many southeastern Asia countries until early May. (4) The decrease in UV and temperature during the coming austral winter can contribute to the sharp increase in South America. For example, both the confirmed and dead cases in Brazil ranked second in the world since 13 June.

The current study provides epidemiological support for the hypothesis that the ultraviolet radiation and air temperature drives the COVID-19 transmission ${ }^{26}$. Our results also implicate a seasonality of COVID-19 and provide an independent measure of the incubation period. The virus transmits more readily during winter and during the season of global monsoon, which impacts about $70 \%$ of the global population ${ }^{34}$. Accordingly, we predict a high possibility of a resurgence in the next boreal winter and suggest to adapt the public policy according to the seasonal variability. 


\section{Methods: Daily growth rate of COVID-19 cases}

The current section extracts a daily infection growth rate for each country from the data of confirmed cases, through a sliding window regression, optimization, cross-correlation, and unit conversion.

\section{A sliding window regression for describing the evolution of the outbreak}

The early stage of an uncontrolled outbreak is characterized by an exponential growth with time, e.g. ${ }^{11,14}$. As an example, Figure S1a displays the cumulative confirmed case number $y(t)$ as a function of time $t$, which follows the exponential law largely. Therefore, we fit the $y(t)$ to an exponential model $y=a e^{b(t-\tau)}$ in a 28day-wide sliding window. (Note that the conclusions of the current work are qualitatively not subject to the window size here, learn from the same analyses but with different window sizes from 16 to 60 days, which are not displayed here.) Here, $\tau$ denotes the center of the sliding window, $a$ measures the confirmed cases at $\tau$, and the exponent factor $b$ measures the growth rate. Measuring the goodness of the regression is $r^{2}$, which is equal to the square of the correlation coefficient between $y(t)$ and its regression value. A low $r^{2}$ represents the growth does not follow the exponential law well. We repeat the regression at $\tau=2,4, \ldots, 170$ days yielding $a$ and $b$ as functions of $\tau$. Displayed in Figure S1b is $b(\tau)$ for Afghanistan as an example.

Implementing the sliding window regression for all countries results in $a(\tau), b(\tau)$, and $r^{2}(\tau)$ for all countries. Scattered in Figures S2a and S2b, are $a(\tau)$ and $r^{2}(\tau)$ against $b(\tau)$ for all countries, respectively. We exclude the $b$ values associated with $r^{2}<0.9$. At $r^{2}<0.9, b$ exhibits a dependence on $r^{2}$, which could be explained in terms of the three Phases (in "Results"). In Phase III and as a response to the artificial controllers, the growth stagnates and does not follow the exponential law anymore.

\section{An optimization for extracting the least-constrained growth rate}

In principle, all the regressed growth rates $b$ values from all countries could be used for correlation analyses. However, for a given country, the regressed $b$ values are not completely independent of each other due to the overlapped sampling associated with our sliding window. Therefore, in the current subsection, we select only one regressed growth rate, denoted as $b_{m}$, for each country. We select $b_{m}$ as the maximum $b$ at $a>\theta_{a}$ where $\theta_{a}$ is a threshold value. $\theta_{a}$ is optimized by maximizing the absolute correlation coefficient $\left|r\left(\theta_{a}\right)\right|$ between $b_{m}\left(\theta_{a}\right)$ and the corresponding meteorological parameters. The coefficients for the UV flux and temperature $\left|r_{U V}\left(\theta_{a}\right)\right|$ and $\left|r_{T}\left(\theta_{a}\right)\right|$ are displayed in Figure S3 as functions of $\theta_{a} \cdot\left|r_{U V}\left(\theta_{a}\right)\right|$, greater than $\left|r_{T}\left(\theta_{a}\right)\right|$, maximizes at $\theta_{a}=2500$. The red crosses in Figure S2a denote $b_{m}$ at $\theta_{a}=2500$, namely, the maximum $b$ at $a>2500$. Most of these crosses are between $2500<a<3000$, suggesting the growth is modulated statistically strongest by the meteorological conditions when there are about 2500-3000 confirmed cases. As an example, the red symbols in Figure S1 illustrate the determined $b_{m}$ and the associated time window.

\section{A cross-correlation for diagnosing the incubation period}

Note that the correlation analyses above are implemented with a time displacement between the sampling window of the growth rate and that of the meteorological variables $\delta t:=t_{U V, T}-\tau$, to avoid the contamination from the COVID-19 incubation period, e.g., ${ }^{18}$. We diagnosis the incubation period through cross-correlation analyses.

We first calculate the absolute correlation coefficient $\left|r_{U V}\right|$ between 28d-averaged UV flux and $b_{m}$ as a function of the displacement $\delta t$. The resultant $\left|r_{U V}(\delta t)\right|$ is displayed as the solid blue line in Figure 3. The slope of the blue line $d\left|r_{U V}\right| / d \delta t$ is denoted as the red crosses. At $-7 \mathrm{~d}<\delta t<0$, the slope $d\left|r_{U V}\right| / d \delta t$ is beyond its standard deviation (outside the shadow), reflecting the correlation decrease sharply as the $\delta t$ increases. We attribute this sharp decrease to the overlapping of the incubation period with the 28d-wide UV simpling window. Note that the identification of the incubation period is not subjective to the threshold $\theta_{a}$, which is learned from the same analyses but with different $\theta_{a}=10^{3.1}, 10^{3.2}, \ldots, 10^{3.8}$ (not displayed here).

The dotted line and bars display the correlation between $b_{m}$ and temperature, and its slope, which does not exhibit a similar sudden drop.

\section{Conversing the growth rate into percentage}

According to our regression model, $b_{m}$ is an exponent and $e^{b_{m}}$ measures the ratio of the regressed number of confirmed cases of one day over that of the previous day. Therefore, $g:=\left(e^{b_{m}}-1\right) * 100 \%$ is the daily growth rate by percentage. When $b_{m} \approx 0$, $b_{m}$ is already a first-order approximation of $g$ due to $g=b_{m}+\mathscr{O}\left(b_{m}^{2}\right) \approx b_{m}$, since $e^{b}$ can be expanded into Taylor polynomial $e^{b}=\sum_{n=0}^{\infty} \frac{b}{n !}=1+b+\mathscr{O}\left(b^{2}\right)$. Here, $\mathscr{O}\left(b^{2}\right)$ denotes a variable with absolute value at most some constant times $\left|b^{2}\right|$ when $b$ is close enough to 0 . In "Results", the analyses are based on $g$. 
medRxiv preprint doi: https://doi.org/10.1101/2020.10.13.20183111; this version posted October 14, 2020. The copyright holder for this preprint (which was not certified by peer review) is the author/funder, who has granted medRxiv a license to display the preprint in perpetuity.

It is made available under a CC-BY-NC 4.0 International license .

\section{Acknowledgments}

This study was funded by the National Science Foundation of China (41888101, 41822101 and 41971022), Deutsche Forschungsgemeinschaft (DFG HE6915/1-1), Strategic Priority Research Program of the Chinese Academy of Sciences (XDB26020000), the State Administration of Foreign Experts Affairs of China (GS20190157002), fellowship for the National Youth Talent Support Program of China (Ten Thousand People Plan). Support from the Swedish Formas (Future Research Leaders) project is also acknowledged. We used the COVID-19 data of cumulative confirmed cases until 20 July of 2020 at a country level from COVID-19 Data Repository by the Center for Systems Science and Engineering (CSSE) at Johns Hopkins University. The daily meteorological variables are extracted from the ERA5 reanalysis dataset from the European Centre for Medium-Range Weather Forecasts (ECMWF) (C3S, 2017). The meteorological variables analyzed herein include the air temperature at $2 \mathrm{~m}$ above the surface (land, sea or inland waters), precipitation, relative humidity, wind speed at the height of $10 \mathrm{~m}$, downward UV radiation flux at the surface (UV, in the range $250-440 \mathrm{~nm}$ ), and diurnal temperature range. The daily mean meteorological data were averaged for each country to compare with the country-level COVID-19 data.

\section{References}

1. Killerby, M. E. et al. Human coronavirus circulation in the united states 2014-2017. J. Clin. Virol. 101, 52 - 56, DOI: https://doi.org/10.1016/j.jcv.2018.01.019 (2018).

2. Shaman, J., Pitzer, V. E., Viboud, C., Grenfell, B. T. \& Lipsitch, M. Absolute humidity and the seasonal onset of influenza in the continental united states. PLOS Biol. 8, 1-13, DOI: 10.1371/journal.pbio.1000316 (2010).

3. Shaman, J. \& Kohn, M. Absolute humidity modulates influenza survival, transmission, and seasonality. Proc. Natl. Acad. Sci. 106, 3243-3248, DOI: 10.1073/pnas.0806852106 (2009). https://www.pnas.org/content/106/9/3243.full.pdf.

4. Schuit, M. et al. Airborne SARS-CoV-2 Is Rapidly Inactivated by Simulated Sunlight. J. Infect. Dis. 222, 564-571, DOI: 10.1093/infdis/jiaa334 (2020).

5. Ratnesar-Shumate, S. et al. Simulated Sunlight Rapidly Inactivates SARS-CoV-2 on Surfaces. J. Infect. Dis. 222, 214-222, DOI: 10.1093/infdis/jiaa274 (2020).

6. Ujiie, M., Tsuzuki, S. \& Ohmagari, N. Effect of temperature on the infectivity of COVID-19. Int. J. Infect. Dis. 95, 301-303, DOI: 10.1016/j.ijid.2020.04.068 (2020).

7. Yao, Y. et al. No association of COVID-19 transmission with temperature or UV radiation in Chinese cities. Eur. Respir. J. 55, 2000517, DOI: 10.1183/13993003.00517-2020 (2020).

8. Qi, H. et al. Covid-19 transmission in mainland china is associated with temperature and humidity: A time-series analysis. Sci. The Total. Environ. 728, 138778, DOI: https://doi.org/10.1016/j.scitotenv.2020.138778 (2020).

9. Sajadi, M. M. et al. Temperature, Humidity, and Latitude Analysis to Estimate Potential Spread and Seasonality of Coronavirus Disease 2019 (COVID-19). JAMA Netw. open 3, e2011834-e2011834, DOI: 10.1001/jamanetworkopen.2020. 11834 (2020).

10. Iqbal, M. M. et al. The effects of regional climatic condition on the spread of COVID-19 at global scale. Sci. Total. Environ. 739, 140101, DOI: 10.1016/j.scitotenv.2020.140101 (2020).

11. Maier, B. F. \& Brockmann, D. Effective containment explains subexponential growth in recent confirmed COVID-19 cases in China. Sci. (80-. ). 368, 742-746, DOI: 10.1126/science.abb4557 (2020).

12. Picoli Junior, S. d. et al. Spreading patterns of the influenza a (h1n1) pandemic. PLOS ONE 6, 1-4, DOI: 10.1371/journal. pone.0017823 (2011).

13. Hunt, A. G. Exponential growth in ebola outbreak since may 14, 2014. Complexity 20, 8-11, DOI: 10.1002/cplx.21615 (2014). https://onlinelibrary.wiley.com/doi/pdf/10.1002/cplx.21615.

14. Liu, Y., Gayle, A. A., Wilder-Smith, A. \& Rocklöv, J. The reproductive number of COVID-19 is higher compared to SARS coronavirus. J. Travel. Med. 27, DOI: 10.1093/jtm/taaa021 (2020).

15. Anderson, R. M., Anderson, B. \& May, R. M. Infectious diseases of humans: dynamics and control (Oxford university press, 1992).

16. Lai, S. et al. Effect of non-pharmaceutical interventions to contain COVID-19 in China. Nature DOI: 10.1038/ s41586-020-2293-x (2020).

17. Tian, H. et al. An investigation of transmission control measures during the first 50 days of the COVID-19 epidemic in China. Sci. (80-. ). 368, 638-642, DOI: 10.1126/science.abb6105 (2020). 
medRxiv preprint doi: https://doi.org/10.1101/2020.10.13.20183111; this version posted October 14, 2020. The copyright holder for this preprint (which was not certified by peer review) is the author/funder, who has granted medRxiv a license to display the preprint in perpetuity.

It is made available under a CC-BY-NC 4.0 International license .

18. Lauer, S. A. et al. The incubation period of coronavirus disease 2019 (CoVID-19) from publicly reported confirmed cases: Estimation and application. Ann. Intern. Med. 172, 577-582, DOI: 10.7326/M20-0504 (2020).

19. Qin, J. et al. Estimation of incubation period distribution of covid-19 using disease onset forward time: A novel crosssectional and forward follow-up study. Sci. Adv. 6, DOI: 10.1126/sciadv.abc1202 (2020). https://advances.sciencemag.org/ content/6/33/eabc1202.full.pdf.

20. Khalatbari-Soltani, S., Cumming, R. C., Delpierre, C. \& Kelly-Irving, M. Importance of collecting data on socioeconomic determinants from the early stage of the COVID-19 outbreak onwards. J. Epidemiol. Community Heal. 74, 620-623, DOI: 10.1136/jech-2020-214297 (2020).

21. Guha, A., Bonsu, J., Dey, A. \& Addison, D. Community and Socioeconomic Factors Associated with COVID-19 in the United States: Zip code level cross sectional analysis. medRxiv Prepr. Serv. Heal. Sci. 2020.04.19.20071944, DOI: 10.1101/2020.04.19.20071944 (2020).

22. Cauchemez, S., Valleron, A. J., Boëlle, P. Y., Flahault, A. \& Ferguson, N. M. Estimating the impact of school closure on influenza transmission from Sentinel data. Nature 452, 750-754, DOI: 10.1038/nature06732 (2008).

23. Dowell, S. F. Seasonal variation in host susceptibility and cycles of certain infectious diseases. Emerg. Infect. Dis. 7, 369-374, DOI: 10.3201/eid0703.017301 (2001).

24. Abhimanyu \& Coussens, A. K. The role of uv radiation and vitamin d in the seasonality and outcomes of infectious disease. Photochem. Photobiol. Sci. 16, 314-338, DOI: 10.1039/C6PP00355A (2017).

25. Martineau, A. R. et al. Vitamin d supplementation to prevent acute respiratory tract infections: systematic review and meta-analysis of individual participant data. BMJ 356, DOI: 10.1136/bmj.i6583 (2017). https://www.bmj.com/content/ 356/bmj.i6583.full.pdf.

26. Whittemore, P. B. COVID-19 Fatalities, Latitude, Sunlight, and Vitamin D. Am. J. Infect. Control. (2020).

27. Sagripanti, J. L. \& Lytle, C. D. Inactivation of influenza virus by solar radiation. Photochem. Photobiol. 83, 1278-1282, DOI: 10.1111/j.1751-1097.2007.00177.x (2007).

28. Sutton, D. et al. Inactivation of the infectivity of two highly pathogenic avian influenza viruses and a virulent Newcastle disease virus by ultraviolet radiation. Avian Pathol. 42, 566-568, DOI: 10.1080/03079457.2013.853867 (2013).

29. Abduljalil, J. M. \& Abduljalil, B. M. Epidemiology, genome, and clinical features of the pandemic SARS-CoV-2: a recent view. New Microbes New Infect. 35, DOI: 10.1016/j.nmni.2020.100672 (2020).

30. Gunthe, S. S., Swain, B., Patra, S. S. \& Amte, A. On the global trends and spread of the COVID-19 outbreak: preliminary assessment of the potential relation between location-specific temperature and UV index. J. Public Heal. 1-10, DOI: 10.1007/s10389-020-01279-y (2020).

31. Kudo, E. et al. Low ambient humidity impairs barrier function and innate resistance against influenza infection. Proc. Natl. Acad. Sci. U. S. A. 166, 10905-10910, DOI: 10.1073/pnas.1902840116 (2019).

32. Seber, G. A. F. Multivariate observations, vol. 252 (John Wiley \& Sons, 2009).

33. Wang, B. \& Ho, L. Rainy season of the Asian-Pacific summer monsson. J. Clim. 15, 386-398, DOI: 10.1175/ 1520-0442(2002)015<0386:RSOTAP>2.0.CO;2 (2002).

34. Wang, P. X. et al. The global monsoon across time scales: Mechanisms and outstanding issues. Earth-Science Rev. 174, 84-121, DOI: 10.1016/j.earscirev.2017.07.006 (2017). 
medRxiv preprint doi: https://doi.org/10.1101/2020.10.13.20183111; this version posted October 14, 2020. The copyright holder for this preprint (which was not certified by peer review) is the author/funder, who has granted medRxiv a license to display the preprint in perpetuity.

It is made available under a CC-BY-NC 4.0 International license .

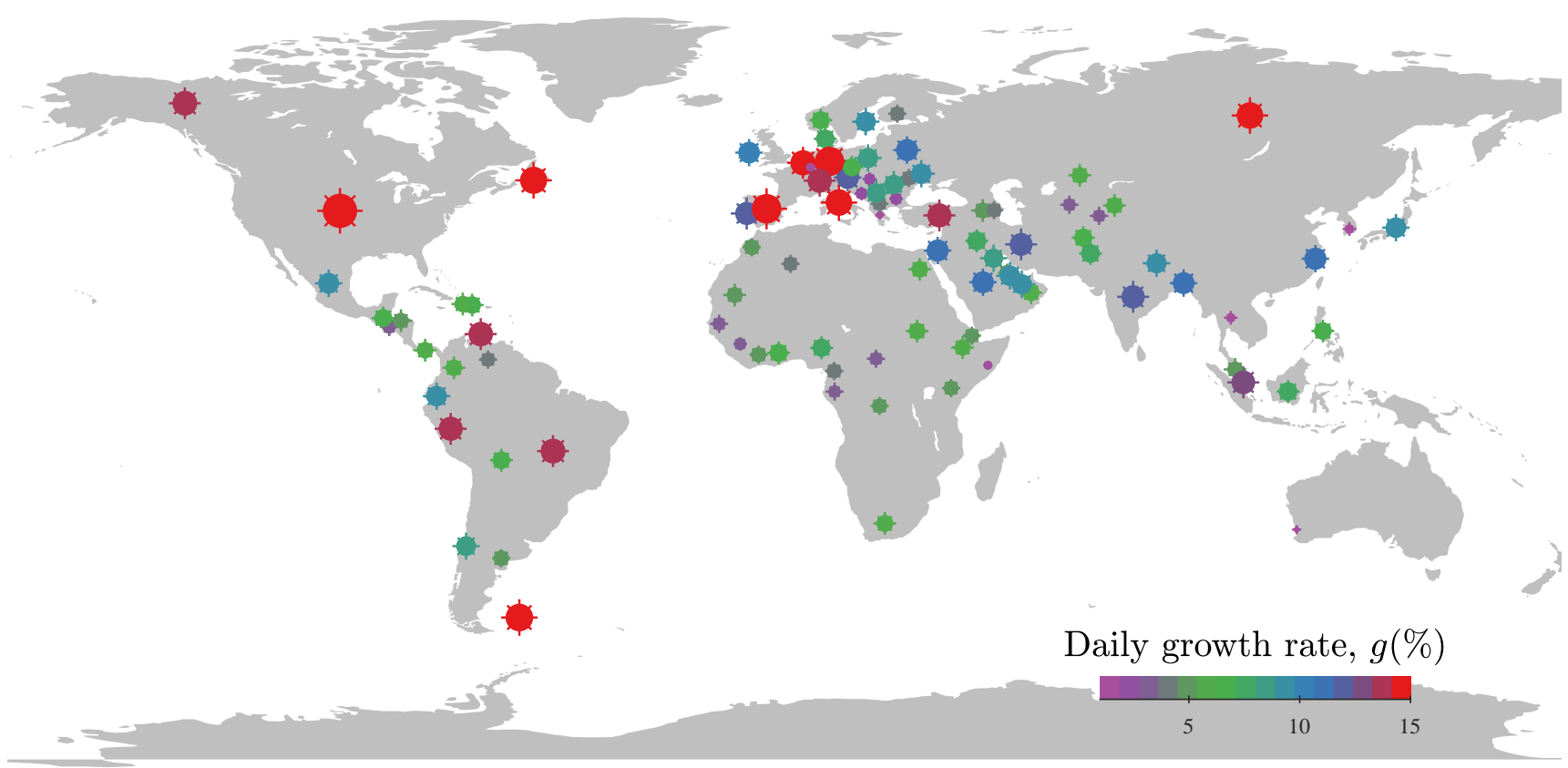

Figure 1. Global distribution of the daily growth rate $g$ of COVID-19 cases. Each point represents one country/region. Both the color and the size of the symbols represent the growth rate. The growth rate is estimated through a sliding window regression and optimization detailed in "Methods". 

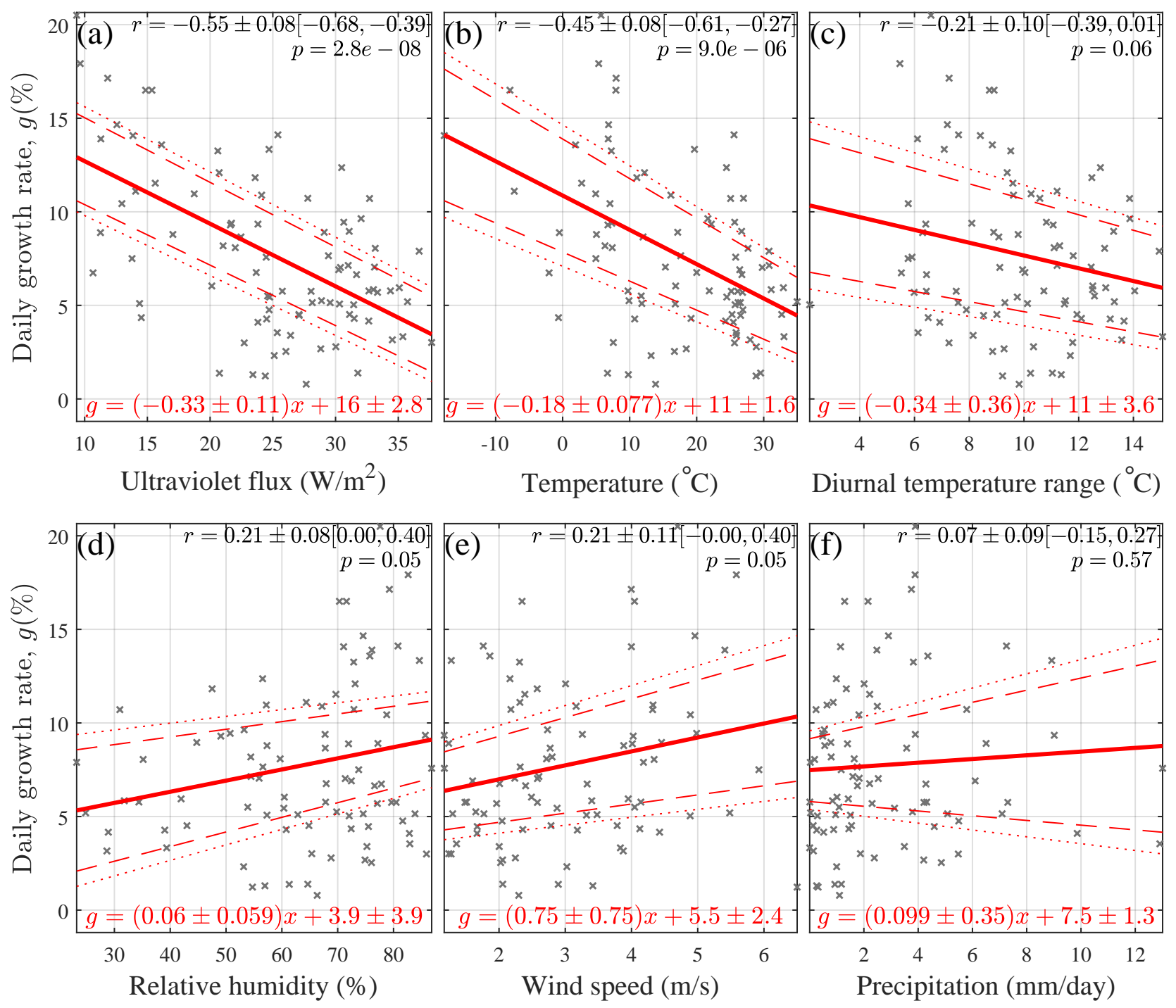

Figure 2. Correlation between the daily growth rate $g$ and six meteorological variables, (a) the ultraviolet (UV) the flux in the range $250-440 \mathrm{~nm}$, (b) the air temperature at $2 \mathrm{~m}$ above the surface, (c) the diurnal temperature range, (d) the relative humidity, (e) the wind speed at the height of $10 \mathrm{~m}$, and (f) the precipitation. In each panel, one cross represents one country, corresponding to one red cross displayed in Figure S2a; the solid red line presents a robust regression to a linear model $g=\beta_{1} * x+\beta_{0}$ through the least absolute deviations method, and the dashed and dotted lines display the significance level $\alpha=$ 0.05 and 0.01 , respectively. Here, $x$ denotes one of the above six variables, and $\beta_{1}$ and $\beta_{0}$ denote the parameters to be determined. The regression results are displayed in red on the bottom of each panel, while the Pearson correlation coefficient $r$ is printed on the upright conner, in the format of $r \pm \Delta r\left[r_{l}, r_{u}\right]$. Here, $r$ and $\Delta r$ are the mean coefficient and its standard deviation estimated through a bootstrapping method, and $r_{l}$ and $r_{u}$ are the lower and upper bounds for a 95\% confidence interval. Also displayed on the top is the $p$-value for testing the hypothesis of no correlation. 


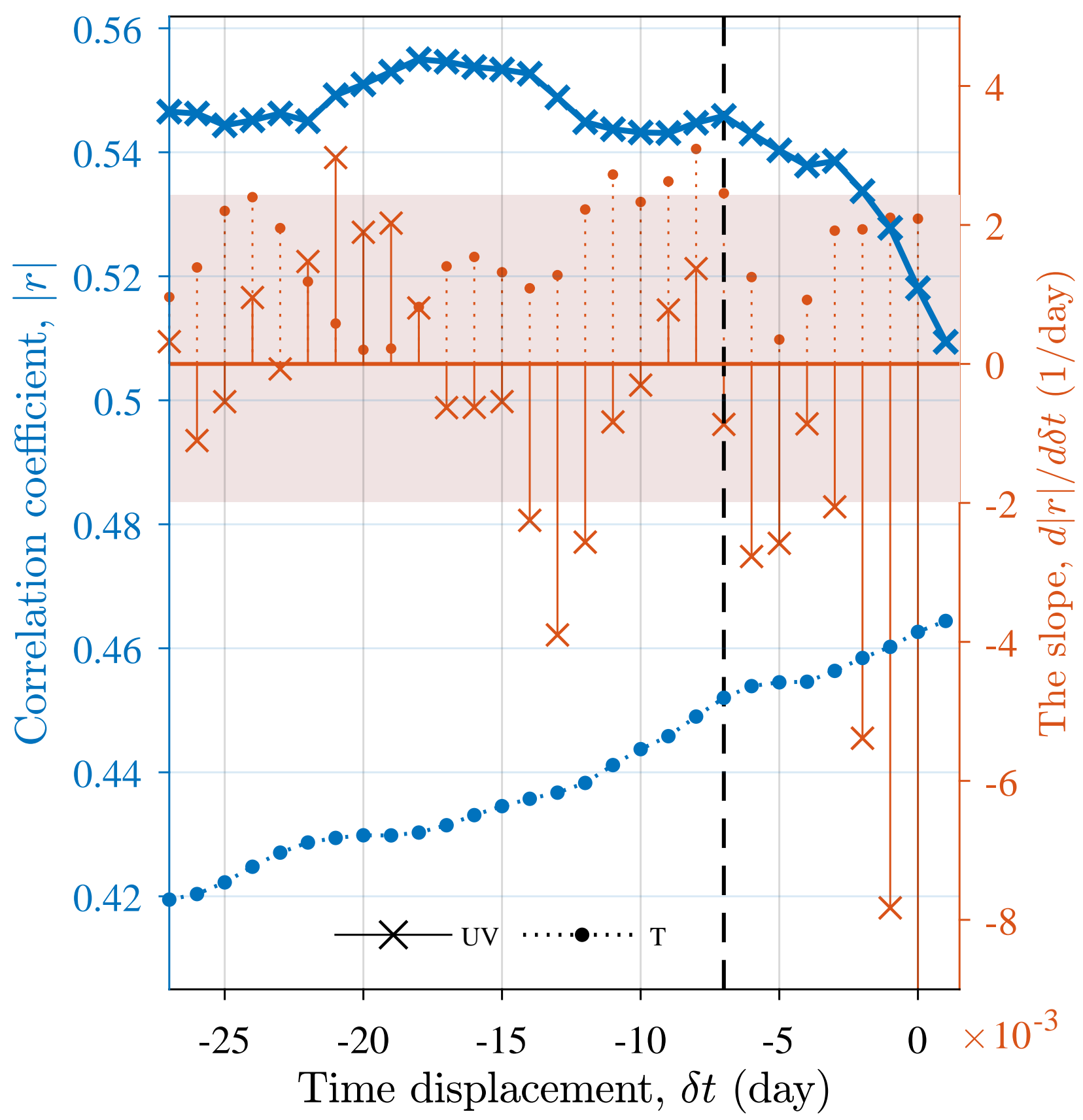

Figure 3. The absolute correlation coefficients $|r|$ of the growth rate with the UV flux and temperature $T$, as functions of the time displacement $\delta t:=t_{U V, T}-\tau$, and their slopes calculated using the centered differencing method. Here, $t_{U V, T}$ is the center of the sampling window of $U V$ or $T$, whereas $\tau$ is the center of the sliding window in which the growth rate is regressed, as instanced in Figure S1. The shadow illustrates one standard deviation of the slopes below and above the average slop. At $\delta t<-7$ as displayed by the black line, the slope of $r_{U V}$ is below the shadow, which is attributable to the incubation period of COVID-19. 